\title{
marges Marges
}

revue d'art contemporain Revue d'art contemporain

\section{Quand un nom vaut des millions. État et limites d'un marché de l'art fondé sur une économie du nom d'artiste}

When a Name is Worth Millions. Status and Limits of an Art Market based upon an Artist's Name Economy

\section{Anne-Sophie V. E. Radermecker}

\section{OpenEdition \\ Journals}

Édition électronique

URL : https://journals.openedition.org/marges/1804

DOI : 10.4000/marges. 1804

ISSN : 2416-8742

\section{Éditeur}

Presses universitaires de Vincennes

\section{Édition imprimée}

Date de publication : 19 avril 2019

Pagination : 44-61

ISBN : 978-2-84292-996-1

ISSN : $1767-7114$

\section{Référence électronique}

Anne-Sophie V. E. Radermecker, «Quand un nom vaut des millions. État et limites d'un marché de l'art fondé sur une économie du nom d'artiste », Marges [En ligne], 28 | 2019, mis en ligne le 01 janvier 2022, consulté le 06 janvier 2022. URL : http://journals.openedition.org/marges/1804 ; DOI : https:// doi.org/10.4000/marges. 1804 


\section{Quand un nom vaut des millions. État et limites d'un marché de l'art fondé sur une économie du nom d'artiste}

/1 Cet article s'inscrit dans le cadre d'une recherche doctorale dédiée à l'économie du nom sur le marché de la peinture ancienne menée grâce au soutien du Fonds National de la Recherche Scientifique de Belgique (F.R.S-FNRS).

/2 Nathalie Moureau, Analyse économique de la valeur des biens d'art, Paris, Economica, 2000, p. 7 et p. 93.

/3 Le nom d'artiste était déjà à l'époque recherché pour lui-même, en adéquation avec les normes de goût ambiant. Voir sur ces questions Koenraad Jonckheere et Filip Vermeylen, « A world of deception and deceit? Jacob Campo Weyerman and the eighteenthcentury art market »,

\section{Introduction}

L'actualité du marché de l'art a coutume de faire la part belle aux records de prix atteints par certains artistes/1, dont les noms - Munch, Bacon, Modigliani, Picasso ou, plus récemment, De Vinci - résonnent non sans une certaine familiarité auprès d'un large public. Lorsqu'il convient en revanche de nommer, voire de visualiser l'œuvre à l'origine du record, la réponse demeure moins évidente alors qu'il s'agit pourtant de l'objet même de la transaction. Cette situation est symptomatique de ce que l'économiste Nathalie Moureau définit comme un marché des noms, dont les prémices remonteraient, selon l'auteure, à l'avènement de l'art moderne/2. Si la quête marchande du nom d'artiste est en réalité attestée dès la première modernité/3, le phénomène n'a eu de cesse de s'accentuer avec désormais un marché de l'art globalisé dont les assises s'apparentent, à bien des égards, à une économie du nom. À l'exception d'une minorité d'artistes, bénéficiant aujourd'hui d'une réputation stable et du statut de valeur refuge, nombre de segments de marché sont en effet contraints de faire face à cette économie singulière. Sur le marché de l'art classé, l'incertitude repose sur l'instabilité du nom, lequel peut être l'objet de réattributions, tandis que sur le marché de l'art contemporain, l'instabilité porte sur les valeurs esthétiques, susceptibles de changer dans un intervalle de temps court et de provoquer la chute rapide 
d'un nom et de sa cote/4. En contrepartie, la médiatisation des records de ventes ainsi que les rankings et autres indices de prix établis à partir des noms les plus lucratifs, concourent à l'édification du nom d'artiste comme valeur centrale du marché, à l'instar d'une logique attributive qui, depuis les débuts de l'histoire de l'art, confère une importance capitale à celui-ci et parfois au détriment des œuvres proprement dites. Acheter de l'art revient ainsi pour bon nombre de personnes à acheter de " grands noms », de " grandes signatures/5 », le nom s'assimilant aujourd'hui davantage à une marque de fabrique qu'à une garantie d'autographie, au sens d'intervention physique de l'artiste dans son œuvre. Se faire un nom, vite et bien, et faire parler de ce nom, procèdent dès lors d'une nécessité presque vitale pour tout artiste et autres acteurs du marché/6, soucieux de s'imposer dans un environnement caractérisé par une forte compétitivité.

Le présent article propose d'interroger le rapport qu'entretient le marché de l'art au nom d'artiste et, plus précisément, à un système prescriptif qui tend de plus en plus à conditionner l'offre et la demande. À l'issue d'un rapide historique permettant de mieux saisir le rapport évolutif du marché à l'autographie et au nom d'artiste, des preuves empiriques du poids du nom sur le marché - en tant qu'identifiant référant à un individu et signe matérialisé dans la signature - sont apportées. À la lumière de ces données, trois axes de réflexion sont développés afin de pointer les tensions que soulève la prescription du nom sur le marché de l'art. Dans un premier temps, le nom d'artiste est pensé comme une construction culturelle, dotée d'une valeur symbolique et marchande instable. Dans un second temps, les effets d'une prescription du nom sur les comportements d'acquisition (acheteurs) et de création (artistes) sont envisagés, avant d'aborder la question des marchés « sans noms », au sein desquels circulent des biens artistiques caractérisés par l'anonymat. L'article invite enfin à relativiser l'importance accordée au nom, en pointant un risque de perte de diversité artistique. Eu égard aux multiples réalités que couvre la notion de négoce de l'art, il convient d'emblée de préciser que la réflexion s'appuie principalement sur des observations faites dans les circuits du marché dit «haut de gamme » ce qui, par conséquent, prévient toute forme de généralisation à l'ensemble du marché.

\section{Une histoire de l'art des noms}

La réception du nom d'artiste sur le marché de l'art ne peut être raisonnablement appréhendée sans une remise en contexte préalable vis-à-vis d'une histoire de l'art elle-même définie à partir du nom d'artiste. La finalité de la discipline étant, par définition, l'étude des
Simiolus, vol. $35, n^{\circ} 1 / 2$, 2011, p. 108; Dries Lyna, « Name hunting, visual characteristics and "New old Masters" : tracking the taste for paintings at 18 th Century auctions ", Eighteenth Century Studies, vol. 46, $\mathrm{n}^{\circ} 1$, 2012, p. 68.

/4 Raymonde Moulin, Le Marché de l'art: mondialisation et nouvelles technologies, Paris, Flammarion, 2003, p. 12 et p. 19.

/5 L'assimilation entre le nom et l'œuvre (acheter un "Renoir », un « Picasso ») est permise une fois qu'un individu a acquis une certaine notoriété grâce à ses œuvres. Son nom peut alors « être utilisé dans un groupe nominal introduit par un article pour désigner ses œuvres ». Voir Marie-Noëlle Gary-Prieur, « Figuration de l'individu à travers différentes constructions du nom propre en français ", Cahiers de praxématique, vol. 27, 1996, p. 6-7.

/6 Don Thompson, The $\$ 12$ million Stuffed Shark. The Curious Economics of Contemporary Art and Auction Houses, Londres, Aurum Press, 2008, p. 9-44. 
/7 Paul Siblot, « De la signifiance du nom propre ", Cahiers de praxématique, vol. 8 , 1987, p. 109-111.

/8 William Grampp, Pricing the Priceless. Art, Artists and Economics, New York, Basic Books, 1989, p. 130-131; p. 165.

19 Claude Lévi-Strauss, La Pensée sauvage, Paris, Agora, 1962, p. 240.

/10 David E. Leary, «Naming and Knowing: Giving Forms to Things Unknown ", Social Research, vol. 62, $\mathrm{n}^{\circ} 2$, 1995, p. 267-298.

/11 Voir notamment Valentine Henderiks, «L'anonymat dans la peinture flamande du XVe siècle. Des maîtres aux noms d'emprunt aux collaborateurs d'atelier ", dans Sébastien Douchet et Valérie Naudet (sld), L'Anonymat dans les arts et les lettres au Moyen Âge, Provence, Presses de l'Université de Provence, 2016, p. 95-105. manifestations artistiques sous toutes leurs formes, l'artiste y occupe légitimement une place centrale, en tant qu'entité individuelle et singulière à l'origine de ces manifestations. L'artiste, comme tout autre individu, est doté d'un nom qui participe à sa définition et qui, outre sa stricte fonction identificatoire, revêt également une fonction taxinomique nécessaire au discours scientifique/7. Si l'attribution d'une œuvre résulte d'une volonté de satisfaire la curiosité humaine pour les objets nommés et identifiés/8, le nom propre permet avant tout la structuration du savoir en favorisant l'identification et la classification de ses objets d'étude/9, d'après la logique qui veut qu'un corpus d'œuvres attribué avec certitude à un artiste est un corpus qui peut être soumis aux méthodes d'analyse historique et scientifique/10. L'attributionnisme, soit la faculté d'assigner des œuvres à un référent (l'auteur), a entre autres permis à l'histoire de l'art d'assurer son développement en tant que discipline scientifique, les œuvres et les noms d'artistes étant à leur tour groupés sous des appellations plus génériques qualifiant les mouvements artistiques. Conférer des noms aux œuvres facilite ainsi le processus d'étude d'un corpus relativement homogène et garantit l'identité (présumée) de l'artiste, en lui rendant les mérites qui lui incombent. Cette structuration de l'histoire de l'art autour du nom a de surcroît largement déterminé les formats de publications et d'expositions inhérents à la discipline. Nombre de dictionnaires d'artistes, de catalogues raisonnés et autres ouvrages monographiques répondent à cette logique classificatoire organisée autour du nom, laquelle est également attestée dans la plupart des catalogues de vente qui à leur tour exploitent ces publications afin de documenter les lots offerts sur le marché. Il convient toutefois de souligner à ce stade que ce processus taxinomique, s'il n'en reste pas moins approprié pour l'art moderne, contemporain et actuel, se heurte à des productions pour lesquelles le nom d'artiste ne peut, à titre temporaire ou définitif, faire l'objet d'une identification précise. Pour des raisons conjoncturelles (anonymat inhérent à l'artisanat, guildes, pratiques d'ateliers/11...) et historiques (pertes ou destructions documentaires...), certaines périodes de l'histoire des arts n'offrent pas toujours la possibilité d'associer une œuvre à un nom. Toujours selon une logique attributive, des solutions ont été élaborées afin de pallier l'absence de noms, lesquelles ont par ailleurs été rapidement récupérées par le marché. L'usage d'une échelle d'authentification, composée de degrés d'attribution dont la valeur décroît à mesure que s'estompe l'authenticité, ainsi que le recours aux noms d'emprunt permettent de conférer une identité indirecte ou fictive à des œuvres pour lesquelles le nom fait défaut. Si l'absence d'autographie provoque une chute indéniable de la valeur marchande 
de l'œuvre/12, ces désignations alternatives permettent néanmoins au marché de gérer une vaste production d'œuvres anonymes avec des retombées marchandes pouvant parfois surpasser celles des œuvres nommées/13.

Il serait toutefois réducteur de faire remonter les origines d'un rapport exacerbé au nom au $19^{\mathrm{e}}$ siècle, époque à laquelle remonte l'institutionnalisation de l'histoire de l'art en tant que discipline scientifique. L'émancipation de l'artiste et la revendication progressive de sa position et de son nom sont en effet à situer dès le Moyen Âge tardif, période marquée par une prise de conscience collective quant à l'importance sociétale de la figure du créateur/14. Les fondements d'une histoire de l'art des noms peuvent, plus spécifiquement, être détectés dans l'historiographie du $16^{\mathrm{e}}$ siècle, sous la plume d'individus déterminés à rendre gloire aux plus grands artistes de leur temps. Des auteurs tels que Giorgio Vasari ou Karel Van Mander/15 ont, par leurs écrits, jeté les prémices d'une histoire de l'art pensée et organisée autour du nom d'artiste et de sa biographie, et selon un dispositif évolutif et hiérarchique qui assurait déjà à l'époque la primauté de certains noms sur d'autres. Très tôt donc se sont distinguées les premières personnalités dites superstars, lesquelles ont bénéficié de témoignages historiographiques suffisamment éloquents pour résister à l'épreuve du temps et ce aux dépens d'autres noms, jugés moins dignes d'intérêt à l'époque. En d'autres termes, la Renaissance contribua à assimiler l'œuvre au nom/16. Il faudra toutefois attendre l'avènement du romantisme au $19^{\mathrm{e}}$ siècle pour que l'importance accordée à l'individualité artistique atteigne son paroxysme. L'exacerbation des notions d'originalité et d'authenticité, ainsi que le culte rendu à la main de l'artiste, ont renforcé l'apologie du génie individuel, lequel revêt un nom dont il est l'incarnation. L'amalgame "œuvre/nom » s'est durablement perpétué avec l'artiste moderne et contemporain dont les patronymes matérialisent, par le biais de corpus d'œuvres relativement homogènes, la vision unique d'un artiste unique, doté d'une identité artistique forte qui, pour être pérenne, se doit d'être isolable et reconnaissable d'entre toutes/17. Le nom tend depuis à fonctionner comme une marque, un label à part entière, qui facilite le processus cognitif d'identification d'une production originale.

\section{La valeur du nom d'artiste sur le marché de l'art}

Si les noms d'artistes permettent aux acteurs du marché de structurer l'offre, c'est principalement sur cette notion de marque que le marché capitalise. Selon un raisonnement marketing, les biens de consommation porteurs d'une marque (ou d'un nom, dans le cas présent) sont
/12 Laura Onofri, « Old Master Paintings, Export Veto and Price Formation: an Empirical Study ", European Journal of Law Economics, vol. 28, 2009, p. 149-161.

/13 C'est par exemple le cas d'une œuvre du cercle de Gérard David, vendue en 2014 pour 11250 euros, alors qu'une œuvre autographe de Crispin van den Broeck ne dépassa pas les 7000 euros la même année.

/14 Sur l'émancipation du statut d'artiste, voir entre autres Martin Warnke, L'Artiste et la cour. Aux origines de l'artiste moderne, trad. S. Bollack, Paris, Éditions de la Maison des Sciences de l'Homme, 1989.

/15 Giorgio Vasari, Le vite de' più eccellenti pittori, scultori, e architettori, Edition Giuntina, 1568; Karel Van Mander, Het Schilder-boek, Haarlem, Passchier Wesbusch, 1604.

/16 Alain Brunn et François-René Martin, «Auteur », Encyclopaedia Universalis, [www. universalis.fr/ encyclopedie/auteur], consulté le 9 février 2018.

/17 Xiyin Tang, « The Artist as Brand: Toward a Trademark Conception of Moral Rights ", The Yale Law Journal, vol. 122, $n^{\circ} 1,2012$, p. 231 et p. 236. 
/18 Jeffrey A. Dubin, " The Demand for Branded and Unbranded Products.

An Econometric Method for Valuing Intangible Assets ", dans Studies in Consumer Demand Econometric Methods Applied to Market Data, New York, Springer, 1998, p. 77-127.

/19 Voir autre autres, Helmut Leder et al., «A Model of aesthetic appreciation and aesthetic judgments », British Journal of Psychology, vol. 95, 2004, p. 489-508.

/20 Benno Belke et al., "When a Picasso is a "Picasso" : the entry point in the identification of visual art ", Acta Psychologica, vol. 133, $\mathrm{n}^{\circ}$ 2, 2009, p. 191-202.

/ 21 Axel Cleeremans, Victor Ginsburgh, Olivier Klein et Abdul Noury, «What's in a name? The effect of an Artist's Name on Aesthetic Judgments », Empirical Studies of the Arts, vol. 34, 2016, p. 126-139.

/ 22 Elisa Hernando et Sara Campo, « Does the Artist's Name Influence the Perceived Value of an Art Work? ", International Journal of Arts Management, vol. 19, $\mathrm{n}^{\circ} 2$, 2017, p. 46-58.

/23 Voir Victor Ginsburgh, Jianping Mei, et Michael Moses, "The computation of price indices ", dans Victor Ginsburgh et David Throsby (sld), Handbook of the Economics of Art and Culture, vol. 1, Amsterdam/ New York, North Holland, 2006, p. 947-979. en général plus aisés à identifier et à promouvoir, à l'inverse de ceux qui en sont dépourvus/18. Le nom de marque s'apparente de surcroît à un label de qualité qui permet de procéder à une différenciation de produits, chaque marque offrant des spécificités propres qui peuvent être perçues différemment d'un consommateur à l'autre. Autrement dit, les noms de marque ont la faculté d'influencer, voire de conditionner certains comportements d'achat sur le marché. Plusieurs études menées dans le domaine des arts plastiques ont, dans une optique similaire, démontré à quel point le nom d'artiste peut déterminer le jugement esthétique porté sur les œuvres/19. Celui-ci sert en effet de point d'entrée au mécanisme de perception et d'appréciation des arts visuels et d'autant plus si le nom peut être associé à un style singulier/20. L'association entre une œuvre non signée et un cartel indiquant le nom de l'auteur permet d'attirer davantage l'attention des spectateurs vers l'œuvre et d'en accroître l'appréciation, notamment si le public est composé de non-experts/21. Pris dans son acception marchande, le nom de marque « exerce une influence aussi bien sur l'évaluation générale de l'œuvre que sur certains aspects spécifiques, tels que sa valeur financière, et le montant d'argent qu'un collectionneur serait prêt à débourser pour l'acquérir/22 ». Le nom de marque contribue ainsi à déterminer la propension à payer des acheteurs, Elisa Hernando et Sara Campo insistant, en guise de recommandations, sur la nécessité de faire la promotion du nom de l'artiste afin d'accroître la valeur perçue de son travail.

L'estimation de la valeur marchande du nom d'artiste est une préoccupation qui, quant à elle, relève davantage des sciences économiques et ce en vue de produire des indices et/ou d'estimer les déterminants de prix sur le marché de l'art. Pour ce faire, les noms d'artistes, traduits en variables binaires, sont intégrés aux modèles économétriques afin de pondérer les indices en fonction de la réputation de chaque nom/23. La prise en compte de cette variable est en effet indispensable à l'appréhension correcte du processus d'estimation des prix sur le marché de la revente/24. La régression hédonistique [Hedonic Pricing Model] est utilisée parmi d'autres méthodes économétriques pour estimer les déterminants de prix sur le marché de l'art. Ce modèle suggère que toute œuvre d'art peut être appréhendée comme un ensemble de caractéristiques observables qui revêtent une valeur implicite équivalant à un pourcentage du prix global de l'œuvre. Dans ce modèle, chaque œuvre vendue est décomposée en un ensemble de caractéristiques intrinsèques et extrinsèques qui participent à la définition de sa valeur marchande (nom de l'artiste, attribution, dimensions, sujet, matériau, technique, signature, date, passages en expositions, publications, salle de vente, année de vente, etc.). Les résultats de 
la régression hédonistique prennent la forme de coefficients, dotés d'une valeur positive ou négative, significativement différente ou non de zéro. Ces coefficients permettent d'estimer le pourcentage qu'il convient d'ajouter ou de retirer au prix moyen des œuvres de l'échantillon d'analyse présentant une caractéristique similaire, en comparaison à un groupe de référence qui ne présente pas cette caractéristique. Si ces études ne publient pas systématiquement les résultats obtenus pour chaque artiste, en raison d'échantillons comptabilisant parfois plusieurs milliers de noms, toutes convergent vers la conclusion que les noms affectent statistiquement et significativement les prix de ventes. À titre d'exemple, l'étude de Dorota Witkowska/25, portant sur les ventes d'artistes polonais entre 2007 et 2010, démontre à quel point les prix moyens atteints par les noms d'artistes de l'échantillon - a priori peu connus du grand grand public - présentent entre eux des écarts différentiels significatifs. Une fois le nom Leon Wyczółkowski (1852-1936) pris comme valeur de référence (100), les résultats de la régression hédonistique montrent que les œuvres des autres artistes sont, en moyenne, entre $11 \%$ et $89 \%$ moins chères, en fonction du nom qui leur est associé :

Leon Wyczółkowski (nom de référence) : 100
Edward Dwurnik: $-11 \%$
Tadeusz Dominik: $-15 \%$
Jerzy Kossak: $-21 \%$
Krynicki Nikifor : $-27 \%$
Władysław Chmieliński : -30\%
Erno Erb: $-34 \%$
Wlastimil Hofman : $-34 \%$
Wojciech Kossak : $-42 \%$
Jerzy Nowosielski : $-89 \%$
Jacek Malczewski : $+36 \%$

Seules les œuvres attribuées à Jacek Malczewski présentent une valeur marchande supérieure, estimée à $+36 \%$. Ces ratios soutiennent ainsi la thèse qui veut que les noms d'artistes induisent des différences de prix significatives sur le marché de l'art. Plus intéressant encore, ces différences ne concernent pas uniquement la dichotomie " grands noms » versus "petits noms », mais se retrouvent également sur les marchés d'artistes plus locaux, indépendamment de facteurs tels que la disponibilité de l'offre et la liquidité des œuvres. Des résultats statistiquement significatifs sont également observés une fois la variable « signature » considérée, laquelle permet de tenir compte de la transcription physique du nom de l'artiste sur son œuvre.
/24 Nauro F. Campos et Renata Leite Barbosa, «Paintings and numbers: An econometric investigation of sales rates, prices, and returns in Latin American art auctions ", Oxford Economic Papers, vol. 61, 2009, p. 31.

/25 Voir Dorota Witkowska, «An Application of Hedonic Regression to Evaluate Prices of Polish Paintings », International Advances in Economic Research, vol. 20, 2014, p. $290-291$.

/26 Luc Renneboog et Christophe Spaenjers, "Buying beauty: on prices and returns in the art market ", Management Science, vol. 59, 2013, p. 36-53; id., " The Iconic Boom in Modern Russian Art », Journal of Alternative Investments, vol. 13 , 2011, p. 67-80.

/ 27 Yi Zhou, « Narcissism and the art market performance", The European Journal of Finance, vol. 23, 2017, p. 1197-1218.

/ 28 Victor Ginsburgh et Nicolas Schwed, « Price Trends for Old Master's Drawings 1980-1991 ", The Art Newspaper, septembre 1992.

/29 Anne-Sophie Radermecker, Victor Ginsburgh et Denni Tommasi, « The Implicit Value of Art Expert. The Case of Klaus Ertz and Pieter Brueghel the Younger ", Solvay-ECARES Working Paper, 2017. 
/30 Nauro F. Campos et Renata Leite Barbosa, op. cit., p. 28; Dorota Witkowska, op. cit., p. 281-293; Corinna Czujack, « Picasso paintings at auction, 1963-1994 ", Journal of Cultural Economics, vol.21, 1997, p. 229-247.

/31 John Stuart Mill, Système de logique déductive et inductive, Paris, Alcan, 1896, p. 35 ; Paula Sjöblom, «A Cognitive Approach to the Semantics of Proper Names ", Onoma, vol. 41, 2006, p. 63-65.

/32 Principe énoncé par Jean-Louis Vaxelaire, "La définition linguistique du nom propre ", Langue française, vol. 190, $n^{\circ} 2,2016$, p. 67.

/33 Michael McKinsey, "Understanding proper names », Linguistics and Philosophy, vol. 33, p. 336.

/34 Jean Molino, « Le nom propre dans la langue », Langages $n^{\circ} 66,1982$, p. 12-13; Gilles Granger, «À quoi servent les noms propres? ", ibid., p. 22.

/35 Voir Gladys Lang et Kurt Lang, « Recognition and Renown : The Survival of Artistic Reputation », American Journal of Sociology, vol. $94, \mathrm{n}^{\circ} 1$, 1988, p. 79.

/36 Entrée " reconnaissance " Dictionnaire informatisé de la langue française (ATILF), [http://atilf. atilf.fr].
Les œuvres signées atteignent en moyenne des prix significativement plus élevés que les œuvres non signées. Plus spécifiquement, Luc Renneboog et Christophe Spaenjers estiment ce ratio à $+31 \%$, à partir d'un échantillon couvrant près de 500 ans de production artistique, contre $+20 \%$ pour un échantillon portant exclusivement sur l'art moderne russe/26. Une augmentation similaire de $+40 \%$ est observée pour des œuvres d'art moderne et contemporain/27, +41 \% pour des dessins flamands anciens/28 et $+63 \%$ pour les tableaux signés du maître Pieter Brueghel le Jeune/29. Ces résultats, qui confirment le poids monétaire de la signature d'artiste dans le processus de formation des prix, varient néanmoins d'un échantillon à l'autre, cet impact pouvant être plus ou moins significatif, en fonction de l'échantillon d'artistes concerné/30.

\section{Le nom comme construction culturelle: une valeur symbolique et marchande relative}

Si la valeur accordée au nom d'artiste se justifie pour des motifs historiques, esthétiques et artistiques, il importe néanmoins de s’intéresser à ses éléments constitutifs. L'un des principaux postulats en linguistique suggère que le nom propre n'existe pas en lui-même ni pour lui-même; pris en tant que signe, un nom n'est qu'une «étiquette vide de sens/31 ». Autrement dit, il n'existe de prime abord aucune différence linguistique entre les noms de René Magritte, Paul Delvaux, Francis Alÿs, Luc Tuymans, ou Thomas Lerooy, tous d'origine belge, si ce n'est une assimilation plus ou moins aisée en fonction de leur agencement orthographique respectif/32. Au même titre, leurs œuvres n'ont pas besoin d'un nom pour exister, ni d'ailleurs pour être nommées. Toute œuvre peut en effet être désignée d'après sa matérialité, sa technique ou son iconographie. Leur nature et leurs finalités n'en restent pas moins similaires/33. Or, ces cinq noms (ou ces cinq marques) ne sont pas équivalents en raison de la fonction sémantique du nom propre qui renvoie à une série d'interprétants, c'est-à-dire de représentation mentale plus ou moins signifiantes qui participent à la définition de sa valeur symbolique et marchande/34. Trois interprétants, vecteurs d'information, en particulier, sous-tendent le nom d'artiste: la reconnaissance, la réputation et la popularité/35. La notion de reconnaissance, qui assure à quelqu'un le fait d'être identifié/36, permet à tout artiste d'être à un moment donné connu ou reconnu. En fonction de son degré de reconnaissance, le nom bénéficie également d'une réputation, soit d'une opinion favorable, défavorable ou inexistante émise à son égard/37, laquelle dépend de la personnalité de l'artiste, de ses activités et de son œuvre/38. Prise dans une acception 
plus large, la réputation d'un artiste se définit également comme «le produit d'un ensemble d'évaluations/39 ». La popularité, soit la façon dont un individu est perçu par un public à un moment donné/40, demeure un interprétant indépendant des deux autres; un artiste reconnu et à la réputation établie n'est en effet pas nécessairement populaire tout au long de sa carrière ni posthumément. En d'autres termes, tout nom bénéficie d'une réputation, d'une reconnaissance et d'une popularité plus ou moins importantes et pérennes qui lui permettront de bénéficier d'une légitimation institutionnelle et marchande et de potentiellement passer l'épreuve du temps, la notion de réputation pouvant de surcroît être liée à une démarche artistique singulière ou à une composition prototypique qui participent à ce processus de légitimation. Ainsi, ce qui distingue les cinq noms précédemment évoqués, ce sont les identités qu'ils définissent, c'est-à-dire deux artistes surréalistes classés, deux artistes vivants établis et un jeune artiste belge prometteur dont l'établissement de la réputation est en cours. Ces degrés variables de réputation, de reconnaissance et de popularité ont par ailleurs des répercussions inévitables sur le marché, comme en témoignent les différences de prix précédemment observées dans l'étude de Dorota Witkowska. Une comparaison entre les indices de plusieurs artistes fait également état de telles différences. L'indice de Magritte illustre une croissance plus importante ces dernières années, avec des valeurs nettement supérieures à celles des indices de Delvaux, d'Alÿs et de Tuymans, le maître du surréalisme figurant parmi les plus réputés, reconnus et populaires de la scène belge/41. L'« attribuabilité/42 » des œuvres du peintre - soit la possibilité d'associer aisément un nom à une idiosyncrasie artistique - demeure centrale dans ce cas de figure, puisque la simple évocation du nom de Magritte permet une identification relativement aisée de son œuvre et génère une multitude de références visuelles, grâce au succès de certains prototypes tels que La Trahison des images, plus connue sous le nom de Ceci n'est pas une pipe (1929) et Le fils de l'homme (1964).

C'est donc plus la sémantique que le nom stricto sensu qui est convoitée et valorisée par le marché, puisque celle-ci est étroitement liée à la notion d'expérience artistique. L'assignation d'un nom à une œuvre permet en effet de satisfaire, en théorie, les notions d'autographie et d'authenticité (en tant qu'engagement auctorial de l'artiste dans son œuvre/43), d'inventio et d'originalité (soit la matérialisation de l'inventivité créative de l'auteur dans une œuvre nouvelle et innovante/44), voire éventuellement de rareté, lorsque la production associée à ce nom est limitée ou soumise à un phénomène de raréfaction. La situation actuelle tend donc à suggérer que seuls les artistes à
/37 Entrée « réputation », dans ibid. Cette définition est également reprise par Lucien Karpik, « Réalité marchande et réputation », Communications $n^{\circ} 92$, 2013/2, p. 121.

/38 Gladys Lang et Kurt Lang, op. cit., p. 86.

/39 En raison du fait que le terme latin reputatio signifie lui-même « évaluation ». Voir Pierre-Marie Chauvin, « La sociologie des réputations. Une définition et cinq questions ", Communications $n^{\circ} 92$, 2013/2, p. 131.

/40 Entrée «popularité ", dans Dictionnaire informatisé de la langue française (ATILF), [http:// atilf.atilf.fr].

/ $4 \mathbf{1}$ Aucun indice pour Thomas Lerooy n'a pu être produit puisque l'artiste est encore principalement actif sur le premier marché et que seuls les résultats de ventes publiques permettent l'élaboration de tels graphiques.

/42 Expression empruntée de l'anglais (attributability) et proposée par Hans J. Van Miegroet, «Creating Attributability with the Five Senses of Jan Brueghel the Younger ", dans Debra Taylor Cashion, Henry Luttikhuizen, Ashley D. West (sld), The Primacy of the Image in Northern European Art, 1400-1700. Essays in Honor of Larry Silver, Leiden/Boston, Brill, 2017, p. 487-499. 


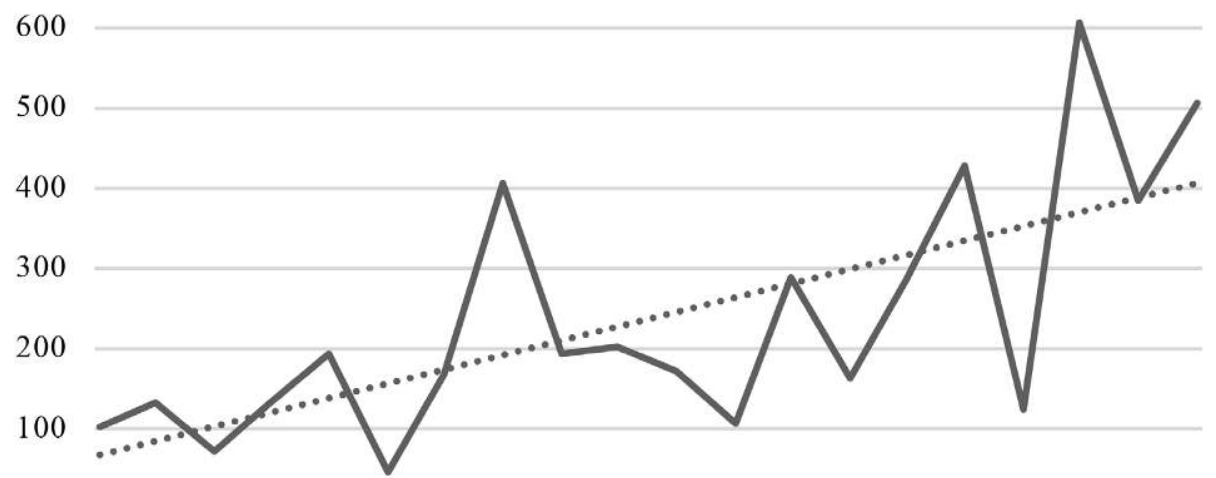

0

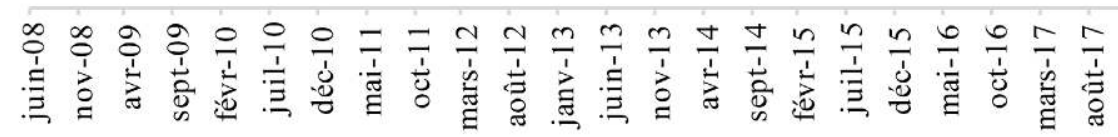

700

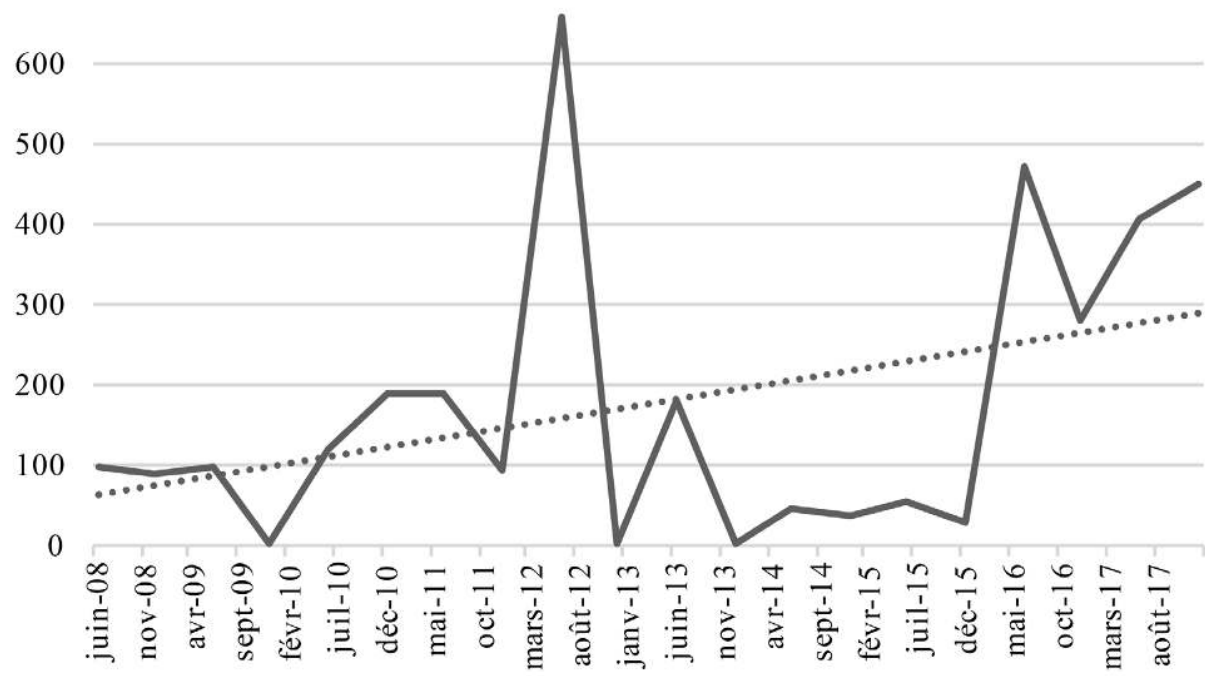

En haut : indice des prix de René Magritte (1898-1967).

En bas : indice des prix de Paul Delvaux

(1897-1994). 
400

350

300

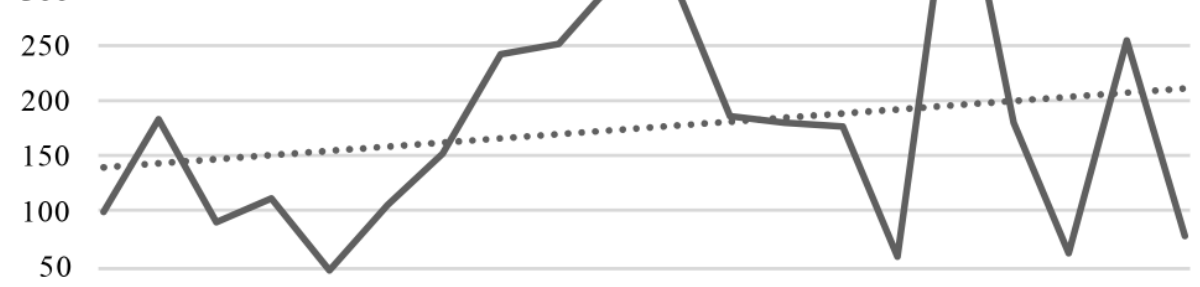

0

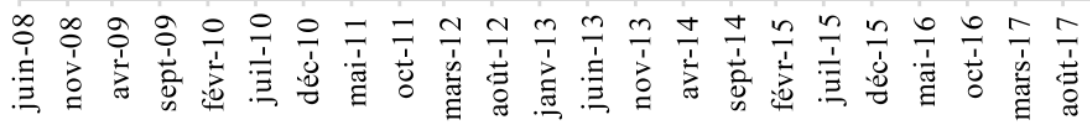

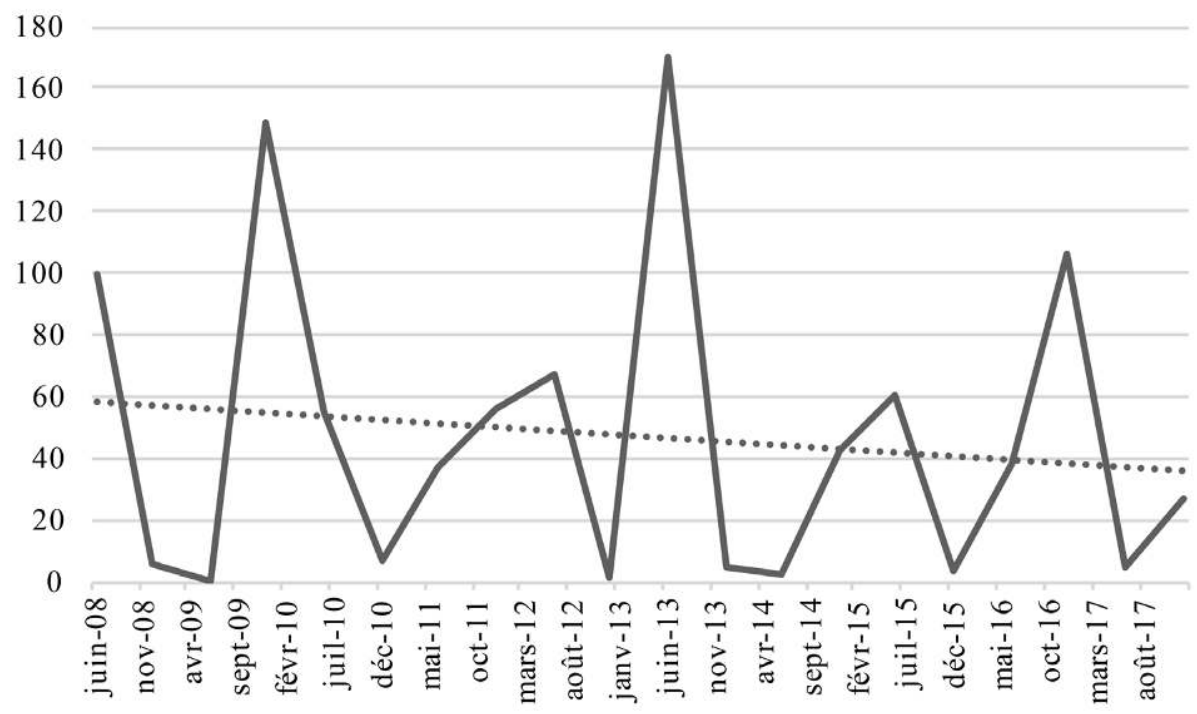

En haut : indice des prix de Francis Alÿs (né en 1959).

En bas: indice des prix de Luc Tuymans (né en 1958). 
/43 Voir notamment à ce sujet Thierry Lenain, "L'authenticité », dans Nathalie Heinich, Jean-Marie Schaeffer, Carole Talon-Hugon (sld), Par-delà le beau et le laid. Enquête sur les valeurs de l'art, Rennes, PUR, 2014, p. 9-22.

/44 Denis Dutton, The Forger's Art: Forgery and the Philosophy of Art, Berkeley \& Los Angeles, University of California Press, 1985, p. 68-71.

/ 45 Certains artistes, à l'instar de Balthus, sont entrés dans l'histoire, mais leur production artistique peut être à l'origine d'une polémique susceptible de ternir leur réputation et leur popularité.

/46 Nathalie Moureau et Dominique SagotDuvauroux parlent de « petits évènements historiques ». Nathalie Moureau et Dominique Sagot-Duvauroux, Le Marché de l'art contemporain, Paris, La Découverte, 2016, p. 78 .

/47 Source: Artprice.com (consulté en mai 2017).

/48 Le mécanisme de légitimation se fait pourtant sur la durée, la notion de réputation pouvant également être corrélée à l'âge de l'artiste. Voir Merijn Rengers et Olav Velthuis, « Determinants of Prices for Contemporary Art in Dutch Galleries, 19921998 ", Journal of Cultural Economics, vol. 26, $\mathrm{n}^{\circ} 1$, 2002, p. 10. la réputation établie sont ceux qui, potentiellement, sont les plus susceptibles de générer des retombées financières significatives, puisque leurs interprétants ont acquis une certaine stabilité. Les autres noms ont, quant à eux, la particularité d'être associés à des interprétants de nature plus instable, lesquels dépendent des normes d'appréciation d'une époque et d'un contexte donnés. L'artiste, de son vivant, a certes une part de responsabilité dans ce processus; ses comportements, son positionnement artistique, sa productivité sont autant de facteurs susceptibles d'influencer sa réputation, sa reconnaissance et sa popularité auprès du public à court, moyen et long termes/45. Néanmoins, ces trois interprétants dépendent également de tierces personnes qui, par leurs actions, leurs discours et leurs réputations respectives/46, peuvent s'avérer favorables ou porter atteinte à la réputation de l'artiste et à l'appréciation de son œuvre. Galeristes, curateurs, critiques, collectionneurs, historiens d'art et experts ont la faculté de déterminer la réception future d'un nom, avec des conséquences parfois décisives sur les cotes d'artistes et de facto sur les comportements d'achat. Or ces mêmes cotes, fréquemment utilisées comme des indicateurs de marché, sont soumises à plusieurs biais d'interprétation qui méritent d'être évoqués. En effet, la plupart des indices de prix prétendent être révélateurs de la réception et de la réputation d'un artiste alors qu'ils témoignent peu de la stabilité et de la pérennité des interprétants du nom. Les chiffres relatifs à l'évolution du marché d'artistes belges depuis 2000 suggèrent, par exemple, que la cote de l'artiste Marcel Broodthaers a accusé une augmentation de $63 \%$ en 2017 , contre $105 \%$ pour Théo van Rysselberghe, $429 \%$ pour Pol Bury, $562 \%$ pour Wim Delvoye et une hausse remarquable de $1103 \%$ pour Walter Leblanc/47. Aussi impressionnants soient-ils (selon une perspective d'investissement), ces chiffres ne peuvent être considérés comme des indicateurs de la réputation historique de ces artistes, et sont susceptibles d'évoluer sur des intervalles de temps courts en fonction de l'évolution de l'offre disponible et de la demande. En outre, les indices actuels produits par des sites d'analyse du marché couvrent généralement des périodes de temps assez courtes, qui témoignent de la valeur moyenne d'un nom y et de ses interprétants sur une période $\mathbf{x} / \mathbf{4 8}$. Ils rendent délicate l'appréciation de son évolution à long terme, les fluctuations annuelles étant plus souvent le fait de facteurs macroéconomiques ou de records de prix, que de changements de goût probants. Les artistes dits «médiatiques/49 " sont révélateurs de cette situation; leur réputation, principalement établie à partir de stratégies spéculatives et non par le biais institutionnel, est caractérisée par une forte instabilité qui leur fait courir le risque de retomber rapidement dans l'anonymat. 
Cette catégorie d'artistes explique d'ailleurs pourquoi les prix sur le marché de l'art ne sont pas toujours proportionnels à la valeur artistique des œuvres. Si ces indices permettent donc d'avoir une idée de la progression d'un nom sur le marché au fil des années, ceux-ci n'en restent pas moins approximatifs et tributaires du mode de sélection des échantillons de ventes analysés. En outre, ils ne reflètent que l'activité d'une minorité d'artistes déjà relativement établis puisque présents sur le second marché.

Le passage à la postérité n'est donc pas synonyme de réception stable et durable; la fortune critique d'un nom peut sensiblement varier en fonction des périodes et du contexte de réception/50. Tout artiste peut donc, à un moment de l'histoire, jouir d'une réputation variable au fil de sa carrière et à titre posthume/51. L'attrait particulier d'un nom et son succès financier sont, en d'autres termes, davantage le fait d'un goût ambiant/52 ou de mouvements spéculatifs orchestrés autour de ce nom, dans le cas des artistes médiatiques, légitimés par le marché mais pas encore par l'histoire de l'art. Or, comme Raymonde Moulin le suggérait déjà, ce sont avant tout les acteurs du secteur institutionnel qui contribuent à assurer la pérennité d'un nom et non ceux du négoce car, à l'instar de tout marché, l'offre et la demande se renouvellent en permanence sous les effets de mode et des changements de critères d'appréciation.

Notons enfin que la réception d'un nom est également fonction du public destinataire. Les noms Magritte et Brueghel sont en effet susceptibles d'être évocateurs auprès d'un public relativement large, et familiarisé un tant soit peu à l'art, puisque tous deux fonctionnent comme des marques artistiques aux idiomes forts et aisément identifiables. En toute probabilité, ceux de Tuymans et Lerooy s'adressent davantage à un public composé de connaisseurs de la scène contemporaine belge établie et émergente. Ce constat contribue donc à renforcer la relativité du nom d'artiste; si certains noms sont signifiants pour un certain public, ces mêmes noms ne vont pas nécessairement de soi pour un public de non-experts, dès lors qu'aucune connexion visuelle ne peut être opérée dans l'esprit du spectateur/53. Il est également permis de penser que, malgré une connaissance du nom de l'artiste et de son imagerie, peu d'individus sont en réalité capables d'identifier physiquement l'individu. Des recherches en psychologie comportementale mériteraient d'être menées afin de tester cette hypothèse. À la lumière de ces éléments, le nom d'artiste peut raisonnablement être pensé comme une construction culturelle susceptible d'évoluer avec le temps. Dans ces circonstances, l'adage qui veut que les petits noms d'aujourd'hui seront les grands noms de demain et vice-versa, conserve toute sa pertinence.
/49 Nathalie Moureau et Dominique SagotDuvauroux, op. cit., p. 22-23.

/50 Voir Francis Haskell, Rediscoveries in Art: Some Aspects of Taste, Fashion and Collecting in England and France, New York, Cornell University Press, 1976.

/51 C'est le cas lors d'événements imprévus, comme le décès de l'artiste, ce qui génère habituellement une hausse sensible des prix. Robert B. Ekelund, Rand W. Ressler et John Keith Watson, " "The Death-Effect" in Art Prices: A Demand-Side Exploration ", Journal of Cultural Economics, vol. $24, n^{\circ} 4,2000$, p. 283300.

/52 Les relations entre goûts et prix de vente sont questionnées dans l'ouvrage magistral de Gerald Reitlinger, The Economics of Taste, 3 vol., Londres, Barrie \& Rockcliff, 1961.

/53 Gérard Petit, « Le nom de marque déposée : nom propre, nom commun et terme ", Meta, vol. 51, $n^{\circ} 4$, 2006, p. 695-699. 
/54 Clare McAndrew, op. cit., p. 16.

/55 Les propos qui suivent concernent toutefois moins les acheteurs de noms émergents, principalement actifs sur le premier marché et pour qui l'appréciation esthétique passe avant la reconnaissance du nom, que le marché de la revente, par lequel transitent des noms plus ou moins établis et dont la production a déjà fait l'objet d'une légitimation institutionnelle et commerciale.

/56 Il importe de reconnaître que, dans certains cas, l'information disponible peut être moindre et la qualité inégale d'un lot à l'autre.

/57 Citons, à titre d'exemples, les galeries Xavier Hufkens (Bruxelles), Rodolphe Janssen (Bruxelles), et Guy Pieters (Knokke).

/58 D'autres voies alternatives telles que les réseaux sociaux permettent d'éviter temporairement les circuits institutionnels et marchands classiques. C'est la stratégie optée par l'artiste espagnol Joan Cornellà qui comptabilise à ce jour plus de 4,5 millions de suiveurs sur Facebook et 1,4 millions de suiveurs sur Instagram, ce qui le positionne parmi les artistes les plus suivis sur les réseaux sociaux. Cette stratégie n'induit pas pour autant une rupture définitive avec le marché, puisque

\section{Les conséquences d'une prescription du nom sur les comportements d'acquisition et de création}

Il existe ainsi une dichotomie entre les grands noms et les noms moins connus, voire inconnus, chacun de ceux-ci faisant l'objet d'une visibilité, d'un intérêt et de gammes de prix variables. Seuls quelques noms permettent de rendre compte de tout un segment de marché, au détriment d'une multitude d'autres noms, émergents, en voie de légitimation ou déjà établis, moins visibles et moins rentables. Les chiffres publiés par le Art Basel Report reflètent explicitement cette situation; en 2016, le secteur des ventes publiques comptabilisait un chiffre d'affaires de 22,1 milliards de dollars, dont près de la moitié n'était le fait que de $1 \%$ des artistes. Seuls $15 \%$ des noms étaient parvenus à accéder au seuil des 50000 dollars, alors que pour la grande majorité des artistes, les médianes ne s'élevaient pas au-delà de quelques milliers de dollars/54. Cette situation de marchandisation du nom d'artiste est par conséquent susceptible d'avoir une série de répercussions sur les comportements d'achat et de création/55.

Du point de vue de la demande, l'examen des catalogues des principales salles de vente permet de faire état d'une structuration de l'offre qui tend à prescrire le nom d'artiste - et certains noms en particulier - auprès des acheteurs, ainsi que l'autographie dans le cas de l'art ancien. Cette prescription se matérialise entre autres dans un discours orienté autour du nom et d'un apport d'information qui diffère notablement d'un lot à l'autre, générant de facto une forte différenciation de produits/56. La mise en valeur de certains noms confère aux salles de vente le pouvoir d'orienter la demande vers ceux jugés les plus attractifs et potentiellement les plus lucratifs à un moment donné et donc d'influencer les comportements d'achat. La promotion inégale des lots lors des ventes tend alors à provoquer une discrimination implicite qui joue en défaveur de certains artistes aux noms certes connus, mais moins populaires et/ou à la réputation moins établie. Sur le premier marché, une autre forme de discrimination intervient dès lors que la carrière d'un artiste émergent dépend étroitement des noms des individus qui en sont les promoteurs. Les artistes belges, aujourd'hui les mieux cotés (Fabre, Borremans, Delvoye...), sont ceux qui, sans surprise, ont transité à un moment de leur carrière dans les galeries les plus influentes du pays/57 et autour desquelles gravitent les collectionneurs les plus nantis et les plus aptes à participer à la création de valeur autour de leurs noms. Autrement dit, le marché contribue à rendre certains noms attractifs auprès des acheteurs, au détriment d'autres noms qui n'ont pas bénéficié des bons réseaux ni des bonnes rencontres et de la visibilité nécessaire à leur émancipation. 
La nécessité de créer un nom qui soit simultanément reconnu, populaire et associé à une imagerie forte, n'est pas non plus sans affecter les comportements de création, si toutefois l'artiste décide d'intégrer le marché de l'art, ce qui relève du libre-arbitre/58. Depuis les années 2000 en particulier, les sciences du marketing se sont intéressées aux stratégies de branding dans le secteur artistique et recommandent depuis aux artistes actuels de se forger un nom de marque et une identité artistique forte, tout en cultivant un mythe autour de leur identité, à l'instar des figures comme Picasso et Warhol/59. Bien que de telles analogies puissent paraître réductrices, le branding est supposé permettre à l'artiste de construire son capital social, culturel et symbolique, selon l'acception bourdieusienne, et de procéder ainsi à sa légitimation sur le marché/6o. Or cette recommandation, présentée comme une clé de succès, peut entrer en contradiction avec la ligne artistique du créateur. Dans un marché de l'art fonctionnant en système relativement fermé, déterminé par les modes, les tendances et le jeu des stratégies spéculatives, l'artiste a le choix de produire en se conformant aux normes esthétiques actuelles et/ ou de se distinguer auprès des acteurs susceptibles d'influencer son marché. Cette exigence de conformisme peut alors constituer un frein à la créativité, puisqu'elle implique un certain formatage de la production, qui consiste à se référer à des modèles préexistants, satisfaisant la demande dans un espace-temps défini. Bien que ce système de référence soit loin d'être le seul fait de l'art actuel - la peinture ancienne étant autrefois régie par les principes d'imitation et d'émulation -, il contraint néanmoins l'artiste émergent à s'adapter aux attentes de la demande, lesquelles peuvent restreindre les possibilités d'actes créatifs libres et autonomes/61. Or c'est précisément cette liberté de création qui permet de mener au renouveau artistique. Ce conformisme est par ailleurs la conséquence d'exigences inhérentes au marché de l'art. Les espaces physiques du négoce (galeries, foires, salles de vente) préconisent, pour des raisons logistiques, certains médiums plus commodes à transporter et à exposer, ce qui derechef provoque une discrimination implicite entre les champs de spécialisation artistique. Certains médiums, formats et techniques sont en effet plus accessibles et commercialisables que d'autres formes d'expression telles que la performance, les installations, le land art ou le video art. En outre, l'artiste ayant établi un contrat avec une galerie se doit, afin d'assurer la visibilité et le rayonnement de son nom, de s'adapter aux échéances du marché et notamment celles des foires qui figurent au calendrier annuel du monde de l'art. Ce contexte de travail, hautement compétitif, n'est pas sans exercer une certaine pression sur l'activité des artistes en voie de reconnaissance. La cadence des certaines de ses œuvres sont mises en vente sur Internet.

/59 Jonathan

E. Schroeder, « The artist and the brand ", European Journal of Marketing, vol. 39, $\mathrm{n}^{\circ} 11 / 12$, 2005, p. 1291-1305; Albert M. Muniz, « Marketing artistic careers: Pablo Picasso as brand manager ", European Journal of Marketing, vol. 48, $n^{\circ} 1 / 2,2014$, p. 68-88; Victoria L. Rodner, «The art of branding - Lessons from Visual Artists ", Arts Marketing: An International Journal, vol. 4, $\mathrm{n}^{\circ} 1 / 2$, 2014, p. 101-118.

/60 Concept développé dans Pierre Bourdieu, La Distinction: critique sociale du jugement, Paris, Minuit, 1979.

/61 Tyler Cowen et Alex Tabarrock établissent une distinction entre les artistes dits « high » et les artistes dits « low », les premiers créant indépendamment des contraintes du marché tandis que les seconds créent en vue de plaire aux attentes actuelles. Tyler Cowen et Alex Tabarrock, «An Economic theory of avant-garde and popular art, or high and low culture ", Southern Economic Journal, vol. 67, $n^{\circ} 2$, 2000, p. 232-253. 
/62 Anne-Sophie Radermecker et Émilie Garcia Guillen, «Le dialogue entre les opérateurs du secteur des arts plastiques contemporains à Bruxelles: perceptions et perspectives d'un territoire créatif », dans Understanding Culture and Creativity in Brussels (à paraître aux VUB-Press, Urban Notebooks series).

/63 Les questions d'attribution et de désattribution ont été au cœur du Rembrandt Research Project (19682011) et d'une exposition au Metropolitan Museum of Art de New York, intitulée « Rembrandt/ Not Rembrandt. Aspect of Connoisseurship ».

/64 Heinrich Wölfflin, Kunstgeschichtliche Grundbegriffe, Munich, Bruckmann, 1915.

/65 Voir à titre d'exemple l'article de Susan Mullin Vogel, «Artists but anonymous works: Fieldwork and Art History ", African Arts, vol. 32, $\mathrm{n}^{\circ}$ 1, 1999 , p. 40-55.

/66 Certains artistes revendiquent leur anonymat afin de se soustraire au diktat du marché. Voir par exemple Cindy Nemser, " Interview with an Anonymous Artist ", Art Education, vol. 23, $\mathrm{n}^{\circ}$ 1, 1970, p. 32-35. L'anonymat intentionnel est souvent associé à une dimension politique. Voir notamment Nicolas Schapira, « Nom propre, évènements est telle qu'elle exige une rapidité de production susceptible d'esquinter le temps de la réflexion inhérent au processus créatif et de porter atteinte à l'innovation et à la qualité. Un risque d'homogénéisation de l'offre et une perte de diversité artistique, au profit d'une rentabilité rapide qui fait fi des exigences du travail créatif, est de ce fait pointé par certains acteurs du monde de l'art/62. L'artiste soucieux de se faire un nom est néanmoins contraint d'adapter son activité à cette dynamique afin d'amorcer la création d'un discours qui participera à la consolidation des interprétants qui sous-tendent son nom en devenir.

\section{Un marché de l'art « sans noms »?}

Si le nom d'artiste peut changer à l'issue de réattributions, disparaître en cas de désattribution, subir une dévaluation ou encore sombrer dans l'oubli en raison de l'imprévisibilité de ses interprétants, l'œuvre d'art demeure la seule garantie de stabilité. Or, les œuvres désattribuées, dépourvues de noms ou aux noms moins familiers tendent à affecter négativement le regard qui leur est porté, ainsi que la propension à payer des acheteurs, alors que leur aspect visuel per se n'en reste pas moins inchangé/63. L'attention accordée au nom d'artiste par le marché tend donc à mettre en retrait l'objet d'art par rapport à son auteur, seule réalité matérielle pourtant possédée in fine par l'acquéreur. Serait-il dès lors légitime d'envisager l'hypothèse d'un marché sans noms dans lequel seule importerait l'œuvre d'art, à l'instar de ce que proposait Heinrich Wölfflin pour l'histoire de l'art/64? De toute évidence, les marchés de l'art sans noms existent, en raison des motifs historiques et conjoncturels évoqués en début d'article, tels que les modes de production d'une époque, les spécificités de l'artisanat et du travail d'atelier, les pertes et les destructions documentaires au fil du temps, etc. Une grande partie des arts non occidentaux/65, des antiquités gréco-romaines, certaines catégories d'arts décoratifs, une part de la production du Moyen Âge tardif et de la Renaissance sont autant de biens qui circulent quotidiennement sur le marché sans qu'aucun nom d'artiste ne puisse leur être assigné. L'art des $19^{\mathrm{e}}, 20^{\mathrm{e}}$ et $21^{\mathrm{e}}$ siècles n'est pas non plus épargné, l'anonymat pouvant être alors intentionnel ou conjoncturel/66. En outre, l'absence de reconnaissance d'un nom peut constituer une forme implicite d'anonymat. C'est en effet ce qui caractérise l'essentiel du marché des chromos, tels que définis par Raymonde Moulin et paradoxalement peu étudiés en raison de leur statut même de "peintures commerciales/67 " ou de la jeune scène émergente dont les noms, bien qu'ils soient disponibles, demeurent implicites au-delà d'un cercle restreint. En conséquence, 
ces segments de marché ont pour caractéristique d'être nettement moins médiatisés, étant donné la rareté des records de vente et la plus faible volatilité des prix. En outre, ils ne permettent pas l'élaboration aisée d'indices et de cotes ce qui, a priori, réduit les possibilités de spéculation et les perspectives d'investissement. L'absence de nom tend donc à réduire la confiance des acheteurs sur un marché déjà caractérisé par une forte asymétrie d'information/68. Cependant, le processus de vente n'en reste pas moins identique, avec des lots présentés dans des catalogues illustrés et documentés lorsque l'information est disponible. Seul l'intitulé du lot diffère; une désignation spatio-temporelle (par exemple: école flamande, $16^{\mathrm{e}}$ siècle; Grèce, $4^{\mathrm{e}}$ siècle av. J.-C.), véritable substitut au nom, permet à l'intéressé de situer l'objet dans le contexte historique auquel les experts estiment qu'il appartient. L'intérêt de ce marché réside donc dans sa capacité à recentrer l'attention sur l'objet stricto sensu, sa matérialité, ses caractéristiques et sa qualité. Cette même notion de qualité n'exclut pas la possibilité d'opérer une différenciation entre des biens de nature similaire, laquelle peut notamment se matérialiser dans les estimations de prix. En d'autres termes, le marché des œuvres anonymes est un marché moins biaisé par des déterminants de prix forts tels que le nom, la réputation et la signature de l'artiste. L'adéquation entre valeur artistique et valeur marchande tend dès lors à être plus optimale, tandis que l'acheteur est quant à lui davantage conscient de ce pour quoi il paie, même si les effets de mode, les erreurs d'authentification ou les faux ne sont pas à exclure de ce segment.

Mais qu'en serait-il d'une vente à l'occasion de laquelle aucun nom ne serait affiché aux côtés d'une sélection d'œuvres non signées? Il est permis de supposer que l'information livrée au spectateur puisse porter en priorité sur l'objet physique: sa technique, son matériau, ses dimensions, le sujet représenté, muni éventuellement de quelques éléments de provenance et de biographie. Le premier contact avec l'œuvre serait, selon toute vraisemblance, marqué par une attention accrue portée à ses qualités matérielles, plastiques et esthétiques. L'anonymat présente en effet l'avantage d'offrir des opportunités d'expériences artistiques autres, reposant sur un jugement plus neutre et non biaisé par les stéréotypes que véhiculent le nom d'artiste et ses interprétants respectifs/69. Mais un tel dispositif présente inévitablement des limites: comment évaluer la valeur effective d'une œuvre privée de nom et en particulier sur le marché de l'art actuel, peu régi par des normes esthétiques spécifiques? En effet, le processus d'estimation des œuvres s'effectue principalement à partir de comparaisons de prix entre des œuvres officiellement assignées à un même nom, tout en tenant compte de multiples événements historiques nom d'auteur et identité sociale. Mises en scènes de l'apparition du nom dans les livres du XVII" siècle", Littératures classiques $n^{\circ}$ 8o, 2013, p. 71; Aldo Milohnic, « How to Do Things with Names and Signatures. On the politics of performatives (re) naming », Performance Research, vol. 22, $\mathrm{n}^{\circ} 5$, 2017, p. 85-93.

167 Raymonde Moulin, Le Marché de la peinture en France, Paris, Minuit, 1967, p. 409-416; Francine Couture, "Le marché des chromos: une industrie culturelle?", Intervention, vol. 12, p. 6-8.

/68 Elisa Hernando et Sara Campo, op. cit., p. 55 .

/69 Konstantinos Vassiliou, «Anonymous art reconsidered: anonymity and the contemporary art institution ", Journal of Aesthetics \& Culture, vol. 9, $\mathrm{n}^{\circ} 1,2017$, p. 1-10. 
/70 Le choix de l'anonymat permet notamment à l'individu d'échapper aux contraintes légales et/ou à la censure sociale. Voir Françoise Zonabend, "Le nom de personne", L'Homme, vol. 20, $\mathrm{n}^{\circ} 4$, 1980, p. 16-17.

/71 Ce principe est attesté par la linguistique et valable pour les noms de convention sur le marché de l'art. Voir Paula Sjöblom, op. cit., p. 70; Kim Oosterlinck et AnneSophie Radermecker, « Master of (...). Creating Names for Art History and the Art Market ", Journal of Cultural Economics, 2018 (à paraître). associés à ce nom. Il est par ailleurs intéressant de s’interroger sur la possibilité d'atteindre un jugement tout à fait neutre avant de procéder à l'acquisition. Même en cas d'anonymat, le nom et le standing de l'intermédiaire de vente peuvent influencer le jugement de l'acheteur puisque certaines galeries sont manifestement plus spécialisées que d'autres dans la promotion de noms établis ou en voie d'établissement. De même, les productions de certains artistes s'apparentent à de véritables marques de fabrique ce qui, même inconsciemment, peut inciter l'acheteur à effectuer des rapprochements comparatifs avec des noms majeurs (par exemple: "à la manière de Duchamp »), à l'instar de ce qui se pratique dans la peinture ancienne lorsque le nom fait défaut. À nouveau, le processus cognitif qui consiste à positionner une œuvre anonyme par rapport à la production d'un nom connu induit en définitive un jugement esthétique qui s'appuie sur ce nom, indépendamment de certaines qualités propres de l'œuvre. L'hypothèse d'un marché sans noms pose également une série d'enjeux pour l'artiste. Le nom permet en effet d'assurer la protection de la propriété intellectuelle et des droits d'auteur or, en l'absence de nom, d'autres alternatives (pseudonymes, noms d'emprunt...) sont susceptibles d'être envisagées/70, lesquelles fonctionnent comme des noms à part entière. Ces alternatives au nom offrent en effet une expérience similaire à celui-ci puisque seule la sémantique importe, comme en témoigne le cas Banksy/71. L'absence de nom peut également constituer un obstacle à la reconnaissance institutionnelle de l'artiste ; comment, en effet, promouvoir et diffuser un corpus d'œuvres produit par un artiste unique sans qu'aucune désignation commune ne puisse les rassembler? Il est probable que des sobriquets ou des noms de commodité soient alors sollicités afin de permettre l'identification du corpus, éviter les erreurs d'attribution et combler l'asymétrie d’information.

\section{Conclusion}

L'objectif de cet article a été de questionner le rapport que le marché, et plus largement une société donnée - soit la société occidentale - entretiennent au nom d'artiste, en envisageant successivement les origines d'une histoire de l'art des noms, la réception du nom d'artiste dans la littérature marketing et économique, ses propriétés linguistiques, ainsi que les implications que cette économie particulière peut avoir sur les comportements d'acquisition et de création. La question des marchés de l'art sans noms a en dernier lieu été posée. En tant que variable instable qui dépend étroitement de trois interprétants (la reconnaissance, la réputation et la popularité), susceptibles 
de fluctuer selon des temporalités variables, le nom d'artiste a été envisagé comme une construction culturelle, soumise aux multiples évaluations opérées par les parties prenantes du monde de l'art. À l'exception de quelques artistes à même de revendiquer une place durable dans le panthéon de l'histoire de l'art, la plupart de ceux présents sur le marché demeurent confrontés à l'instabilité de leur patronyme, avec toutes les conséquences que cette instabilité peut induire sur leur valeur symbolique et marchande. L'économie du nom qui caractérise le marché de l'art actuel tend par ailleurs à faire oublier que la transaction économique qui s'opère entre le vendeur et l'acheteur ne porte pas exclusivement sur le nom ni l'artiste, mais bien sur l'œuvre, en tant que matérialisation de l'expression du créateur. Si les exigences associées à la construction d'un nom et d'une identité visuelle forte peuvent entraver la liberté créative des artistes, la quête marchande du nom, pensée comme une finalité, peut également conduire à une perte de diversité artistique dès lors qu'elle déconsidère tout un pan de la production dépourvue de nom ou aux noms encore peu connus. L'importance conférée au nom d'artiste est néanmoins telle dans le milieu institutionnel et marchand qu'il semble, à ce stade, difficile d'engager un changement radical de mentalité à cet égard. Sans pour autant renoncer aux acquis de la discipline de l'histoire de l'art en matière d'attribution et d'identification, il revient aux recherches futures de proposer des modèles de pensée capables de relativiser le star system actuel qui empêche de nombreux artistes et biens artistico-culturels dépourvus de noms établis, d'être considérés pour leurs qualités propres. Une confrontation de notre vision occidentale de l'art à celle d'autres sociétés (africaines, asiatiques...) semble, à cette fin, offrir un axe de recherche opportun.

\section{Anne-Sophie V. E. Radermecker}

